\title{
Exploring the sustainable development of College Students' autonomous learning ability \\ Maohua SUN
}

\author{
Foreign Language Department, Dalian Jiao Tong University, Liaoning Province, China \\ summer_817@163.com
}

Keywords: autonomous learning; learning ability; sustainable development

\begin{abstract}
This paper discusses how to foster the students' self-learning ability. Through the research on the establishment of the "people-oriented" educational concept, the construction of a new teaching model, and the establishment and improvement of the training mechanism of autonomous learning, the problems caused by the "lack of construction" in the teaching and learning of College English teaching are solved. To foster students' lifelong learning ability and achieve the sustainable development of English learning ability. In order to promote the cultivation and improvement of students' autonomous learning ability, teachers should establish and emphasize humanism teaching ideas, promote the use of all kinds of autonomous learning strategies, guide students to learn the meaning of self building knowledge, learn to learn, learn to study and innovative learning by using various teaching theories, so as to realize learning. It has the ability of self-learning and sustainable development.
\end{abstract}

\section{探究大学生英语自主学习能力的持续性发展}

\author{
孙茂华 \\ 大连交通大学, 大连, 辽宁省, 中国 \\ summer_817@163.com
}

关键词:自主学习;学习能力;可持续发展

中文摘要.本论文探讨了如何促进学生自主学习能力的培养, 通过对建立 “以人为本” 的教育 理念、构建新型教学模式、建立健全自主学习的培养机制等方面的研究, 以解决大学英语教 学 “建构缺失” 状态对教学和学习所造成的问题, 培养学生终身学习的能力, 并以此达到英 语学习能力的可持续发展。要促进学生自主学习能力的培养与提高, 教师应建立并强调人本 主义教学思想, 推进各种自主学习策略的运用, 运用多种教学理论引导学生学会自行构建知 识的意义, 学会学习, 学会进行探究性学习和创新性学习, 从而实现学生自主学习能力的可 持续发展, 并具备终身学习的能力。

\section{1. 建立人本主义的英语教育理念}

\section{1 “以人为本” 的教育理念}

目前, “教育要以人为本”已成为中国教育新发展的重要方针。教育是以培养人为主的 社会活动。人既是教育的出发点也是教育的归宿。人是具体的、活生生的、现实的个体, 教 育以人为本就是要关注学习中的每一位学生的学习活动。 “以人为本” 教育理念的内涵便体 现在尊重和关爱教育主体一一学生的生命本性, 培养和完善学生的社会属性和个性, 其最终 意旨在于关注学生的全面协调、 
持续发展。

罗杰斯的人本主义教育理论倡导教育的终旨是促进学生的自我实现, 目标是使学生的心 理健康得到真正的发展, 并要求教师信任和尊重学生, 提供资源并营造良好的学习气氛。“以 人为本” 教育中的教学思想的核心是教学人性化, 即教学以学生为中心。在中国, 大学英语 教学是大学教学的一个重要组成部分, 大学英语教学改革是教学改革的一个重要环节, 在大 学英语教学中强调 “以人为本” 的教学理念, 符合目前国家对人才需求的要求, 因此是科学 的和与时俱进的。

\section{2 “以人为本” 的英语教学理念}

学生是学习的主体, 也是自身发展的主体, 教师的教只是素质形成和发展的外因, 只有 以学生为主体、以人为本才有助于开发学生的自主性、能动性和创造性。因此, 以人为本的 学生主体观是优化语言课堂教学的价值取向之一, 充分体现了素质教育的本质。英语语言教 学实践性很强, 英语这一文化的载体蕴涵着英语国家悠久的历史、丰富的文化, 传播着来自 世界各地的科技信息。教学中, 学生的语言基础知识、文化背景知识、认知水平、语言能力 等方面千差万别, 教师应充分理解, 并在教学过程中充分运用 “以人为本” 的教育思想, 坚 持 “以学生为中心” 的教学理念, 把 “以人为本” 的教育思想运用到教学中, 满足学生学习 中正当的、合理的需要, 鼓励学生积极投入各种学习活动和交流活动中去, 只有这样, 才能 提高学生的学习兴趣, 使学生真正成为学习的主人, 学生才有可能最大限度地提高自身的英 语语言能力, 汲取西方文化精髓, 并在学习过程中, 养成良好的学习习惯, 获得一定的自主 学习能力, 为口后进一步学习和发展奠定坚实的基础。

\section{2. 构建建构主义的教学模式}

由于旧的教学理念和教学模式不利于创新主义精神以及实践的能力的培养, 建构主义的 理论恰逢其时, 为新的教学模式提供了理论和实践的基础。随着多媒体的计算机和网络教育 应用的飞速发展，在世界范围内口益扩大其影响。

\section{1 建构主义的教学模式}

建构主义学习理论的教学模式是以学生为中心, 教师作为组织者构建适合于建构主义学 习理论的学习环境。在建构主义的教学模式中, 学生作为知识建构的主体参与教学。多媒体 也不再是像黑板一样只作为教师传授知识的唯一载体, 而是成为了教师用来在教学中创设情 境的手段。现代化教育媒体是一种创设真实语言情境的最有效的工具。现代化教育手段能够 有效地帮助英语教师创设出真实具体、栩栩如生的英语学习情境。教师可以根据教学内容的 需要运用体现英语文化的图像和声音等手段创设形式多样的情境, 例如设置一些问题或悬念 启发引导学生联想思维, 或运用一些引人入胜、集知识性和趣味性于一体的场景来激发学生 的学习动机和学习兴趣, 使学生在一种如同真实的体验中利用自己已有知识结构中的相关经 验去同化新学到的知识, 在新旧知识之间建立起联系并赋予某种意义。

以学生为中心的合作学习使小组每一位成员的智慧资源和信息资源就可以被小组全体成 员所共享, 共同完成英语学习和语言技能训练的任务, 以达到对语言知识的意义建构。以课 堂内容为基础的 “英语会话” 训练。要使学生真正成为英语学习的主体即语言信息加工的主 体, 有必要为学生的自主化和个性化学习提供各种各样的方法和信息资源。“英语会话” 学 习会话训练的同时, 在原有的语言知识和语言技能的基础上, 建构新的知识。“英语会话” 训练时, 学生需要一个气氛轻松的课堂环境。运用现代化教学手段能够为学生提供直观、形 象且内容丰富、声情并茂的英语视听材料, 让他们亲身感受真实语言情境、实际操练自己所 学的语言技能并进行意义建构。 
教师、学生、媒体、教材等教学中的四个要素之间有着与过去完全不同的关系。四个要 素之间非常清晰明确的关系, 构成了建构主义学习环境下新型的大学英语教学模式。在新的 教学模式下, 教师必须转变观念。学生英语语言技能的全面发展和个性发展, 才能真正实现 大学英语教学模式的创新与改革。

\section{2 新型大学英语教学模式的建构}

当今社会的发展对大学生的英语实际运用能力提出了更新、更高的要求。教师必须不断 更新大学英语教学理念, 探索适合新形势下的英语教学方法和教学模式的建构。在建构主义 的学习环境之下, 教师、学生之间在教学中的角色和作用都发生了很大变化。基于建构主义 学习理论的教学原则, 新型大学英语教学设计的原则应着重强调 “情境” 意义建构的原则。 对学习 “情境” 的创设是教学设计中最重要的内容之一, 因为在学习中要进行意义建构首先 必须要创设学习情境, 真实、具体且形式、目的各异的学习情境会使学生有身临其境的感觉, 这对他们对目前所学知识的意义进行建构非常有利。在真实、具体的情境中实施自主学习, 学生的认知与元认知能力持续加强。因此, 新型大学英语教学模式的建构应遵循建构主义的 教学原则, 主要表现为:(1>教学的目标应该能与学生的学习环境和学习个性化需求相适应; $(2>$ 教师应预知学生在学习中会遇到的问题并就此设计出相应的学习任务并在课堂的教学过程中 能够使用。真实的教学活动才是学习环境中的重要特征;(3)在教学设计中要能反映出或涉及到 学生们在英语课堂学习之后有能力运用英语应对复杂的交际环境;(4)引导学生自主解决问题, 以激发其创造性思维;(5)引导学生自主监控学习过程, 评估学习效果, 发展他们自主进行和管 理自己学习的能力, 让他们成为能够独立自主学习的人。

\section{3. 培养认知主义的学习策略}

\section{1培养自主学习的认知学习策略}

自主学习的基本特征决定了学生应采取的相应的学习策略。。)那么何谓学习策略?学习 策略是一个多层次和动态的有机系统, 主要分认知策略(cognitivestrategies)和元认知策略 (metacognitive strategies), 二者都以学习方法为基本要素。学习方法是指向理解和指向记忆的 学习知识和技能的最直接的手段, 涉及对学习材料的理解、处理、记忆和提取, 因此学习方 法必须在认知、元认知(metacognition)作用下才能发挥有效作用, 同时又在一定程度上作用于 认知和元认知。

元认知策略是指在第二语言习得中学习者在学习时需使用一些方法和技巧对输入的信息 进行加工处理和控制的过程, 具体到自主学习实际中就是执行自己的学习计划, 安排自己的 学习时间, 监控自己的学习过程, 调节自己的学习步骤, 预测可能会遇到的问题以及相应的 解决办法, 评估和反思自己的学习结果等。运用元认知策略学生可以合理有效地利用现有资 源对接受的学习信息进行处理并作出较为准确的预测和判断, 对自己的学习活动进行计划、 调节、监控和反思, 以达到最佳学习效果。学生要掌握元认知策略, 首先应掌握元认知知识 三个主要方面的内容, 即对个人作为学习者的认识、对自己学习任务的认识、对相关学习策 略及其使用的认识等。因此, 学生的认知知识和认知能力对他们有效使用调节策略具有相当 重要的作用, 能够使用元认知策略进行学习的学生能够意识到自己在学习的某个方面可能出 现的问题, 并及时找出来加以修改, 因此他们的学习是积极的过程。要提高学生自主学习的 能力, 教师就应注重培养学生的元认知能力, 同时有责任教会学生学习和使用元认知策略。 因此, 掌握元认知策略对改善学习效果起着极为关键的作用。

\section{2 内部认知与学生自主能动性培养与发展}

学生的最终学习效果, 个体自身的努力和外部刺激是一方面因素, 而学习个体自身的能 力水平、知识储备、认知方法及非认知的因素构成另一方面的因素。做好学习的学前准备是 
提高学习效果的前提; 要重视反复训练巩固的作用, 因为认知主义学习理论强调学习的主动自 主性, 所以非常重视个体自身动机和在学习过程中逐步形成的强化状态; 强调学习个体自主学 习的创造性, 教师要从培养学生自主学习入手, 充分调动学生学习的积极性, 发挥学生的主 观能动性，教会学生如何学会学习、合作学习与探究学习。

综上所述, 将建构主义学习理论、元认知学习策略等先进的教育教学理念运用于学习实 践之中, 是每一位教师义不容辞的责任, 是每一位渴望成功的学生必须的基本要素之一。运 用元认知策略培养学生英语自主学习能力, 可以是学生在进行自主学习的同时, 对自己的学 习效果进行反思和评估。学生对元认知知识的了解和掌握, 也有利于养成良好的学习习惯, 确保提高学习效率, 形成较强的自主学习能力。因此, 具备元认知策略和技能是具有自主学 习能力的保障。

总之, 元认知策略的培养对于提高大学生英语自主学习能力有着极大的推动作用。因此, 教师在口常英语教学中应该注重学生的元认知策略知识的灌输, 从而切实提高大学生的自主 学习能力和英语综合应用能力。

\section{References}

[1] Du Shi Zhong. "Humanistic education theory" M]. Nanjing: Jiangsu Education Press, 1999

[2] Jiang Rong. The loss and return of human nature. Rodgers's humanistic psychology. M]. Wuhan: Hubei Education Press, 2000.

[3] Li Yuqiu. Principles and applications of psychology. M]. Beijing: Economic Science Press, 1999.

[4] Li Xiaowen, Tu Ying. Teaching strategy "M]. Beijing: Higher Education Press, 2000.

[5] Liang Ningjian. Contemporary cognitive psychology "M]. Shanghai: Shanghai Education Press, 2004.

[6] Chen Jian Lin. The organic integration of computer network and foreign language curriculum under the new mode of College English teaching. An ecological study of the concept of foreign language teaching $\{\mathrm{J}\}$. foreign language audio-visual instruction, 2006 (12): 3-10.

[7] "Chen Qingsong and $\mathrm{Xu}$ Luo." web-based foreign language learning in College English Teaching "J\}. foreign language community, 2006 (6): 16-23.

[8] Cheng Xiao Tang. On white master learning [J\} . discipline education, 1999 (9): 32-39.

[9] "Cheng Xiaotang, Zheng Min. English learning strategies" [M]. Beijing: foreign language education and Research Press, 2002.

[10] Dong Qi. On Metacognition \{J\}. Journal of Beijing Normal University, 1989 (1).

[11]] "Fan Ye." learner training "in College English teaching $\} \mathrm{J}\}$. foreign language field, 1999 (3): 32-37. 